Revue des études hébraïques et juives

\title{
Entre éternité et contingence : la Loi chez Maïmonide
}

Une étude comparée du Guide des égarés et du Livre de la connaissance

Between Eternity and Contingency: Maimonides Conception of Law. A

Comparative Study of the Guide for the Perplexed and The Book of

Knowledge

\section{David Lemler}

\section{OpenEdition}

\section{Journals}

Édition électronique

URL : https://journals.openedition.org/yod/671

DOI : 10.4000/yod.671

ISSN : 2261-0200

Éditeur

INALCO

\section{Édition imprimée}

Date de publication : 1 janvier 2010

Pagination : 57-91

ISBN : 978-2-85831-183-5

ISSN : 0338-9316

Référence électronique

David Lemler, « ENTRE ÉTERNITÉ ET CONTINgENCE : LA LOI CHEZ MAÏMONIDE », Yod [En ligne], 15 | 2010, mis en ligne le 07 octobre 2011, consulté le 08 juillet 2021. URL : http://journals.openedition.org/yod/ 671 ; DOI : https://doi.org/10.4000/yod.671

Ce document a été généré automatiquement le 8 juillet 2021.

\section{(i) (8)}

Yod est mis à disposition selon les termes de la Licence Creative Commons Attribution - Pas d'Utilisation Commerciale 4.0 International. 


\title{
Entre éternité et contingence : la Loi chez Maïmonide
}

\author{
Une étude comparée du Guide des égarés et du Livre de la connaissance \\ Between Eternity and Contingency: Maimonides Conception of Law. A \\ Comparative Study of the Guide for the Perplexed and The Book of \\ Knowledge
}

David Lemler

Cette réflexion se propose de donner un éclairage sur un problème interne à la pensée de Maïmonide que Shlomo Pinès expose et résout de la manière suivante :

Il est clair qu'au point de vue strictement logique une explication qui, pour rendre compte de la raison d'être de nombreux commandements, doit faire appel aux contingences historiques de l'époque de Moïse ne s'accorde pas trop avec le postulat qui nie, pour l'avenir comme pour le passé, toute possibilité d'une abrogation ou d'une modification légitime de n'importe quelle partie de la Torah. D'autre part, il est évident que Maïmonide considère qu'il est obligé de maintenir ce postulat, du moins en droit, car, à son point de vue, la survie du judaïsme est à ce prix ${ }^{1}$.

Le problème est le suivant. Maïmonide use, principalement dans le Guide des égarés ${ }^{2}$, d'une argumentation de type historique pour rendre compte de la motivation rationnelle de certains commandements de la Torah. Il y affirme par ailleurs le caractère immuable de la même Torah, qu'il va jusqu'à postuler dans le Mishneh Torah ${ }^{3}$. Cette contradiction semble dessiner une opposition entre l'entreprise «philosophique » du Guide et celle « halakhique» ou «juridique» du Mishneh Torah, y compris sa partie la plus spéculative, son premier volume, le Sefer ha-madda (Livre de la connaissance).

L'objet de cet article est de montrer que ce paradoxe ne traduit pas une dualité de la doctrine maïmonidienne de la Loi, afin de le délier dans un sens différent de celui, essentiellement politique, proposé par Pinès. Cette bipolarité de la Loi, datée historiquement d'une part, éternelle de l'autre traduit une conception originale du statut de la Torah dans l'œuvre de Maïmonide. Chacun des pôles du problème est à situer dans l'économie de chacune des œuvres: le postulat de l'éternité de la Loi est au 
fondement même de l'entreprise du Mishneh Torah, tandis que le recours à l'argument " historique " s'inscrit dans le projet du Guide d'une démonstration du caractère divin de la Torah. Mais malgré ces différences concernant le fondement de la Torah, Maïmonide propose bien une doctrine unitaire de la Loi. Si la Torah a revêtu une forme déterminée par les contingences historiques de l'époque où elle a été donnée, si certaines de ses dispositions complètement liées à ces contingences, notamment le culte sacrificiel, sont inapplicables en l'absence de Temple, elle n'en vaut pas moins pour les tous les temps, y compris les temps messianiques. Maïmonide construit ainsi un concept de loi divine : est divine une loi qui vaut indépendamment des conditions de son application.

\section{La question des fondements de la Loi}

Le Mishneh Torah et le Guide diffèrent essentiellement par leurs démarches et leurs approches de ce que Maïmonide appelle les «fondements de la Loi». Le premier se contente de les expliciter et de les postuler lorsque le second s'efforce d'en donner une démonstration. C'est à ce niveau qu'apparaît notre contradiction.

\section{Mishneh Torah : l'immutabilité de la Loi comme principe}

La perpétuité et l'immutabilité de la Loi occupent une fonction absolument centrale dans la théorie de la prophétie décrite dans le Mishneh Torah. Cette théorie figure dans les Hilkhot yesodei ha-Torah (règles relatives aux fondements de la Loi), la première section du Sefer ha-madda' consacrée aux principes fondamentaux de la religion. Déjà, parmi les fameux «treize articles de la foi », exposés dans l'introduction au Pereq Heleq du Commentaire sur la Mishna, œuvre de jeunesse de Maïmonide, l'interdiction de rien ajouter ni retrancher à la Torah ( $9^{\mathrm{e}}$ principe) succède à la croyance à la prophétie ( $6^{\mathrm{e}}$ principe), à l'exceptionnalité de la prophétie de Moïse ( $7^{\mathrm{e}}$ principe) et au caractère révélé de la Torah ( $8^{\mathrm{e}}$ principe $)^{4}$. Dans le Sefer ha-madda ${ }^{c}$, le rapport entre ces différents «principes» est précisé et explicité. Maïmonide y décrit d'abord les qualités nécessaires au prophète (chapitre VII). Il s'agit essentiellement de qualités morales et spéculatives, la sagesse, la domination des passions, la méditation constante sur Dieu (halakhah 1), mais aussi de qualités physiques, il est par exemple nécessaire au prophète d'être en bonne santé pour être à même de consacrer son âme à la méditation, afin de recevoir la prophétie. Mais Maïmonide s'empresse de préciser que ces qualités, conditions nécessaires à la prophétie, n'en sont pas des conditions suffisantes (halakha 5) :

Ce sont les hommes qui cherchaient à se mettre en état de prophétiser que l'on nommait les fils des prophètes. Il pouvait se faire que, malgré leurs efforts pour disposer leur entendement à la prophétie, la shekhinah (présence divine) résidât en eux ou non ${ }^{5}$.

Se pose alors la question des critères de reconnaissance de la prophétie authentique. Rien dans la personne d'un individu qui se dit prophète, ni ses caractéristiques physiques, ni ses caractéristiques morales ou spirituelles, ne permet d'établir la vérité de sa prophétie. Se penchant plus spécifiquement sur la prophétie de Moïse dont il vient d'affirmer qu'elle en est le degré ultime - puisqu'à la différence de tous les autres prophètes, Moïse recevait la prophétie en état de veille et non en songe, qu'il la recevait directement de Dieu et non par l'intermédiaire d'un ange, qu'il la recevait, par ailleurs, sans éprouver de tremblements convulsifs, " comme un homme parle à son prochain » 
$($ Ex 33,11$)$ - Maïmonide exclut les signes et les miracles comme critères de validation de la prophétie.

Israël n'a pas cru en Moïse, notre maître, à cause des signes qu'il a accomplis. S'en rapporter au témoignage des signes, c'est, en effet, laisser place en son cœur au doute que le signe a peut-être été exécuté par un enchanteur ou un magicien. [...] Et par quoi Israël fut déterminé à croire en Moïse ? Par la scène du mont Sinaï (ma'amad har Sinaï) : car ce sont nos yeux, et non ceux d'un étranger, qui ont vu, ce sont nos oreilles, et non celle d'un autre, qui ont entendu le feu, les voix et les éclairs. Lorsque Moïse approcha de la nuée ténébreuse et que la Voix lui parla, nous l'entendîmes personnellement lui enjoindre: «Moïse, Moïse va leur parler de telle ou telle sorte." Comme le déclare le prophète lui-même: "C'est face à face que vous a parlé le Seigneur » (Dt 5, 4). De même, il est écrit : "Ce n'est point avec nos pères que le Seigneur a conclu cette alliance, [c'est avec nous qui sommes ici aujourd'hui tous vivants]. » $(D t 5,3)^{6}$.

La scène du Sinaï est ici interprétée comme une scène originelle qui inaugure une communauté nouvelle par le biais d'une révélation directe de Dieu au peuple. Les enfants d'Israël ont entendu de leurs propres oreilles la «Voix» divine, attestant indubitablement la prophétie de Moïse. Maïmonide va encore plus loin, s’incluant dans ce nous présent au mont Sinaï. Si tout Juif doit se considérer le soir de Pesah (Pâque) comme étant lui-même sorti d'Égypte (selon le texte de la Haggadah) ${ }^{7}$, Maïmonide nous invite ici à penser que tout Juif a littéralement assisté à la scène du Sinaï. Les Juifs sont donc autant de "témoins " (selon le mot de Rambam dans la halakha suivante) ${ }^{8}$ de la prophétie de Moïse. Si un homme quel qu'il soit venait à nier la vérité de cette prophétie, le peuple tout entier pourrait témoigner contre lui :

C'est pourquoi si un prophète surgit et accomplit de grands signes et prodiges tout en cherchant à démentir le message prophétique de Moïse, notre maître, en infirmant sa prophétie, nous ne l'écoutons pas, car nous savons alors à l'évidence que ces signes ont été obtenus par les charmes et les prestiges de la magie. En effet, l'authenticité de la mission prophétique de Moïse n'était pas fonction de signes [...], mais nos propres yeux l'ont constatée et nos propres oreilles l'ont entendue comme Moïse lui-même?

La prophétie de Moïse, qui constitue le texte de la Torah, apparaît ainsi comme une métaprophétie fournissant un critère d'authentification des prophètes ultérieurs. Les signes et les prodiges produits par un individu se disant prophète ne sont pas en soi des preuves de la vérité de sa prophétie. Seul celui qui a des caractéristiques morales et spirituelles le rendant apte à la prophétie et qui, de plus, n'infirme en rien la Loi de Moïse peut légitimement être supposé prophète. C'est uniquement s'il répond à ces deux critères préliminaires qu'on lui demandera de produire un signe pour prouver la vérité de sa prophétie. La prophétie de Moïse, elle, ne nécessite aucune preuve prodigieuse, puisque sa vérité est connue avec certitude, pour tous ceux qui étaient présents au Sinaï. Il n'y a pas de place pour le doute dans le cas de Moïse et donc aucune exigence de preuves ${ }^{10}$.

Reste le cas d'un individu, qui serait pourvu des qualités nécessaires à la prophétie, se réclamerait de la prophétie de Moïse et soutiendrait la vérité de sa Loi, tout en affirmant la caducité de certaines ou de toutes ses dispositions. C'est pour contrer une telle figure ${ }^{11}$ que Maïmonide précise :

C'est une notion clairement explicitée dans la Torah que cette dernière reste d'obligation éternellement et dans les siècles des siècles, sans être sujette à subir aucune variation, retranchement, ni complément. Le verset déclare, en effet: "Toutes les choses que je vous prescris, vous les observerez en les mettant en pratique, sans y rien ajouter et sans en retrancher rien.» (Dt 13,1). [...] Il en découle qu'aucun prophète n'y saurait plus désormais introduire la moindre innovation. C'est pourquoi si un homme s'élève provenant d'Israël ou 
des Nations et qu'après avoir produit un signe et un prodige, il prétend que Dieu l'a dépêché pour ajouter ou pour retrancher un commandement ou encore pour donner de l'un quelconque d'entre eux une interprétation que nous n'avons pas reçue de Moïse; ou s'il soutient que les commandements auxquels Israël est astreint ne sont pas éternellement valables et n'intéressent pas toutes les générations, mais qu'ils n'ont été imposés que pour un temps, cet homme est assurément un faux prophète (nevi' sheqer) ${ }^{12}$.

Maïmonide convoque le principe de la perpétuité de la Loi de Moïse, principe énoncé dans la Torah elle-même, dans une perspective nettement polémique, à l'égard du christianisme et de l'Islam. Cela ressort de manière explicite de la première section de l'Épitre au Yémen. ${ }^{13}$ Il est alors tentant d'interpréter l'affirmation de l'immutabilité de la Loi, avec Shlomo Pinès, dans la lignée de son maître Léo Strauss, comme s'inscrivant dans une perspective essentiellement politique. Maïmonide affirmerait l'immutabilité de la Loi, malgré le caractère manifestement " archaïque " à ses yeux, de certaines de ses dispositions, dans le but de sauvegarder la communauté juive, que ce soit simplement parce qu'il est un Juif soucieux de préserver sa propre communauté « nationale », ou parce que, en tant que philosophe, il reconnaît dans la Torah la loi de la communauté idéale, imaginée avant lui par Al-Fârâbîn ${ }^{14}$.

Quoi qu'il en soit, l'immutabilité de la Torah, sa validité pour toutes les générations postsinaïques, est établie dans le Mishneh Torah comme un principe fondamental. À partir du moment où la prophétie de Moïse et par conséquent la Torah est authentifiée par une révélation directe de Dieu au peuple, tous les commandements figurants dans la Torah acquièrent force de loi. L'interdit de rien ajouter ou retrancher à la Torah en fait partie. De manière indirecte, le principe de l'immutabilité est donc établi par la révélation sinaïque, il n'a pas à être prouvé. Qu'il figure parmi les fondements de la Torah (yesodei ha-Torah) ne saurait nous surprendre: il est l'un des présupposés principaux de la démarche même du Mishneh Torah qui est une répétition complète de la Torah orale, une compilation et une codification, inédite dans l'histoire du judaïsme, de la Halakha ${ }^{15}$. Maïmonide, contrairement à la plupart des décisionnaires ${ }^{16}$, tranche, en effet, dans tous les domaines de la Loi, y compris ceux qui n'avaient pas plus d'applicabilités à l'époque de Maïmonide qu'à la nôtre (les lois relatives au service du Temple ou à la royauté par exemple ${ }^{17}$. La loi d'Israël, c'est donc la Torah et toute la Torah, qu'elle soit applicable ou non. Cela engage évidemment une conception de la loi, définie en deçà ou au-delà de son effectuation, qu'il s'agira d'élucider plus pleinement.

\section{Le Guide des égarés : l'argument historique comme preuve du caractère divin de la Torah}

La démarche mise en œuvre dans le Guide part de prémisses absolument différentes, voire opposées. Loin d'être un principe fondamental, un présupposé, le caractère parfait, immuable, pour le dire d'un mot, divin, de la Loi de Moïse y devient problématique. Le « doute » qui était d'office exclu dans le Mishneh Torah, eu égard à la prophétie de Moïse, s'est installé dans l'esprit du destinataire du Guide.

Certes, lorsqu'il dresse le portrait de ce destinataire dans l'avant-propos dédicatoire, Maïmonide précise que :

[ce traité] a pour but de donner l'éveil à l'homme religieux chez lequel la vérité de notre Loi est établie dans l'âme et devenue un objet de croyance, qui est parfait dans sa religion et dans ses mœurs, qui a étudié les sciences des philosophes et en connaît les divers sujets, et que la raison humaine a attiré et guidé pour le faire entrer sur son domaine, mais qui est embarrassé par le sens extérieur (littéral) de la Loi ${ }^{18}$. 
La lecture du Guide, selon les intentions de son auteur, semble donc présupposer la croyance en la vérité de la Loi. Mais l'embarras qu'éprouve le destinataire quant au «sens extérieur de la Loi » paraît indiquer que cette croyance se trouve fortement ébranlée. Il est possible de lire ce passage comme une biographie intellectuelle du destinataire. Celui-ci a d'abord reçu, par éducation et par habitude, la croyance en la vérité de la Loi, puis il a étudié les « sciences des philosophes ». C'est alors qu'un conflit est apparu en lui entre la raison et la Loi, du moins dans son « sens extérieur », et, avec l'apparition de ce conflit, un doute s'est installé quant à la vérité même de cette Loi.

Conformément aux intentions explicites du Guide, il s'agira alors pour Maïmonide de rétablir fermement cette croyance, voire de la faire passer du statut de simple croyance à celui de certitude. Mais cette «certitude » ne saurait se réduire à la certitude sensible issue de la révélation sinaïque qui est au fondement de la démarche du Mishneh Torah. Une des tâches avouées du Guide est, au contraire, d'amener son lecteur à la certitude rationnellement fondée de la vérité de la Loi, de sa perfection et de sa perpétuité.

Il est intéressant à cet égard de comparer le traitement de la validation de la prophétie dans le Guide à celui que nous avons vu à l'œuvre dans le Mishneh Torah. La prophétie apparaît comme un phénomène relevant de la cosmologie et de la psychologie, à travers sa description en termes d'émanations et de facultés :

Sache que la prophétie, en réalité, est une émanation de Dieu, qui se répand [...] sur la faculté rationnelle d'abord, et ensuite sur la faculté imaginative; c'est le plus haut degré de l'homme et le terme de la perfection à laquelle son espèce peut atteindre, et cet état est la plus haute perfection de la faculté imaginative ${ }^{19}$.

Maïmonide reprend ici dans une large mesure la théorie des " qualités nécessaires » à la prophétie présente dans le Mishneh Torah, en l'explicitant davantage et en lui donnant une tournure nettement plus philosophique, s'inspirant fortement de la psychologie et de la cosmologie des péripatéticiens arabes. La prophétie apparaît ici chez Maïmonide comme une véritable union ${ }^{20}$ de Dieu, du moins de son émanation, avec le prophète, du moins ses facultés. L'idée de Maïmonide, dans le cadre de la théorie de la prophétie, est que le prophète ayant accédé à la perfection de la faculté rationnelle et de la faculté imaginative devient apte à recevoir une vision prophétique, qui n'est rien d'autre qu'un « épanchement », une « émanation » divine sur son âme.

Comme l'indique Maïmonide dans une présentation quasi typologique, la perfection rationnelle sans la perfection imaginative est l'apanage des « savants qui se livrent à la spéculation $»^{21}$, tandis que la perfection de la faculté imaginative sans celle de la rationnelle se trouve chez les « hommes d'État qui font les lois, les devins [...] et ceux qui font des songes vrais $»^{22}$. Seule la conjonction des deux perfections permet la vision prophétique authentique. Mais, dans le Guide de même que dans le Mishneh Torah, Maïmonide affirme que la possession de ces perfections facultaires ne suffit pas à l'accès à la prophétie. Dieu seul, par son épanchement, fait passer la prophétie, dans un individu, de la puissance à l'acte. C'est d'ailleurs, selon Maïmonide, ce qui différencie au sujet de la prophétie «l'opinion de notre Loi » de «l'opinion des philosophes », selon laquelle tout homme apte à la prophétie y accède nécessairement ${ }^{23}$.

Comme dans le Mishneh Torah, les caractéristiques internes au supposé prophète ne suffisent pas à attester sa prophétie et, comme dans le Mishneh Torah, la production de miracles ne saurait être à elle seule la preuve de l'authenticité d'une prophétie, puisque dans la catégorie des individus pourvus d'une perfection imaginative, mais dépourvus de perfection rationnelle se trouvent également «ceux qui font des miracles par des 
artifices extraordinaires et des arts occultes, sans pourtant être des savants $»^{24}$. Mais à ce stade, le Guide s'écarte de la théorie de la prophétie élaborée dans le Mishneh Torah, puisque ce que nous avons appelé la "métaprophétie de Moïse ", qui fournit dans le Sefer ha-madda' l'ultime critère d'authentification de la prophétie, n'est plus attestée au peuple par une révélation divine.

Aux chapitres II, 32 et 33, Maïmonide propose, en effet, une réinterprétation minimaliste de la scène du mont Sinaï (máamad har Sinaï). Dans le Mishneh Torah, sa description, comme révélation au cours de laquelle le peuple tout entier a entendu la Voix (qol) de la même façon que Moïse, semble absolument incompatible avec la théorie des qualités nécessaires à la prophétie, puisqu'elle suppose que même les ignorants au sein du peuple aient pu accéder à une vision prophétique. La scène du Sinaï ne peut devenir garantie de la métaprophétie mosaïque qu'au prix de cette incohérence. À son lecteur supposé philosophe, Maïmonide offre au contraire, dans le Guide, une interprétation de la révélation sinaïque qui s'inscrit parfaitement dans la théorie de la double perfection facultaire. Certes, la scène du Sinaï est décrite localement comme une vision prophétique ${ }^{25}$, mais Maïmonide précise par ailleurs qu'elle n'a pas élevé tous les enfants d'Israël au rang de prophètes ${ }^{26}$.

Il est clair pour moi que dans la scène du mont Sinaï, tout ce qui parvint à Moïse ne parvint pas dans sa totalité à tout Israël. [...] Eux, ils entendirent la Voix forte, mais ils ne distinguèrent pas les paroles ${ }^{27}$.

Cette scène constitue bien une révélation directe au peuple entier, mais, contrairement à ce qu'affirmait Maïmonide dans le Mishneh Torah, ce que les enfants d'Israël entendirent est sans commune mesure avec ce qu'entendit leur maître et prophète. Ce qu'ils ont perçu est une pure voix indistincte, une parole sans discours, une adresse sans contenu. Se référant à une tradition midrashique, Maïmonide fait cependant la concession suivante : le peuple dans son ensemble a pu entendre directement de Dieu, mais de manière confuse, les deux premières des dix paroles ('aseret ha-devarim), à savoir «Je suis l'Éternel ton Dieu» et «Tu n'auras pas d'autres dieux que moi », que Maïmonide contrairement à d'autres, comprend comme des commandements et non de simples assertions ${ }^{28}$. Néanmoins, cela n'est concevable à ses yeux que parce que ces deux " commandements », contrairement aux huit suivants, se réduisent précisément à l'énoncé de vérités spéculatives, que tout homme est à même d'atteindre par le biais de la raison et qui ne nécessitent donc pas de révélation prophétique :

En effet, ces deux principes, je veux dire l'existence de Dieu et son unité, on les conçoit par la (simple) spéculation humaine; et tout ce qui peut être su par une démonstration l'est absolument au même titre par le prophète et par tout autre qui le sait ${ }^{29}$.

D'une certaine manière, entendre la pure Voix de Dieu, c'est être contraint, c'est se voir prescrit, de croire en son existence et en son unicité. Ainsi peut-on comprendre l'idée apparemment contradictoire d'une perception confuse de principes rationnels. Ces principes (existence et unicité) sont pensés par Maïmonide sur un mode prescriptif, précisément parce que les hommes n'en ont en général qu'une idée confuse. Les prescrire comme des mișwot, cela revient à les rendre accessibles à tous, y compris à ceux qui n'y auraient jamais eu accès par l'usage de la seule raison.

Par cette réinterprétation de la scène du Sinaï, la théorie maïmonidienne de la prophétie gagne en cohérence, mais en renonçant à l'idée d'une révélation commune au prophète et au peuple, Maïmonide renonce par là même à la certitude immédiate de la vérité de la "métaprophétie » de Moïse. Dans le Mishneh Torah, la prophétie de Moïse était attestée par une révélation qui ne laissait aucune place au doute. L'origine divine 
de la Loi était donc absolument indubitable. Dans le Guide, celle-ci reste à prouver. Au chapitre II, 40, traitant de la nature de la loi en général, Maïmonide énonce à son destinataire un programme : celui de lui fournir

un critérium au moyen duquel tu puisses faire la distinction entre les régimes de lois conventionnelles, ceux de la loi divine et ceux émanés d'hommes qui ont fait des emprunts aux paroles des prophètes, en s'en vantant et en se les attribuant ${ }^{30}$.

\section{La démonstration du caractère divin de la Loi}

La prophétie de Moïse disposait, dans le Mishneh Torah, d'un critère d'authentification extérieur, celui de la révélation divine au Sinaï. Dans le Guide, nous ne disposons plus d'un tel critère extérieur et celui-ci doit être cherché à l'intérieur même du texte prophétique. Cela impose de procéder à un examen de la Loi de Moïse pour vérifier qu'elle répond bien aux critères définissant une loi divine. Ces critères sont décrits plus loin dans le même chapitre :

Mais, si tu trouves une loi dont toutes les dispositions visent (non seulement) à l'amélioration des intérêts corporels, dont on vient de parler, mais aussi à l'amélioration de la foi, s'efforçant tout d'abord de répandre des opinions saines sur Dieu et sur les anges, et tendant à rendre l'homme sage, intelligent et attentif, pour qu'il connaisse tout l'être selon sa vraie condition, alors tu sauras que ce régime émane de Dieu, et que cette loi est divine. Mais il te restera encore à savoir si celui qui la proclame est lui-même l'homme parfait auquel elle a été révélée, ou si c'est une personne qui s'est vantée de ces discours et se les est faussement attribués. - Pour en faire l'expérience, il faut examiner (jusqu'où va) la perfection de cette personne, épier ses actions et considérer sa conduite ${ }^{31}$.

La présomption du caractère divin d'une loi provient de ses caractéristiques internes et non des circonstances éventuellement miraculeuses dans lesquelles elle a été donnée : cette loi doit à la fois prescrire des normes purement politiques, visant «à mettre en bon ordre l'État et ses affaires et à en écarter l'injustice et la violence ${ }^{32}-$ tels sont les "intérêts corporels" dont parle ici Maïmonide, qui sont donc essentiellement à comprendre comme les intérêts du corps politique - et inculquer des vérités spéculatives, relatives à Dieu. Il sera alors clair que l'homme édictant cette loi ne vise pas uniquement à s'approprier un pouvoir temporel. Une fois établi, par une analyse interne, le caractère potentiellement divin d'une loi, il faut encore s'assurer que l'homme qui la proclame est bien un homme apte à la prophétie, en examinant ses qualités psychologiques et morales. S'il s'avère que cet homme répond aux exigences nécessaires à la prophétie, alors seulement, il sera établi que la loi qu'il édicte est bien d'origine divine.

C'est donc dans le cadre de la théorie de la prophétie du Guide, que s'inscrit la nécessité de passer la Torah au crible de la raison. Il s'agit de montrer que toutes les dispositions inscrites dans la Torah visent bien l'un des deux objectifs de toute loi divine : assurer le bien-être du corps individuel comme politique (faire cesser la violence réciproque et donner de "bonnes mœurs ») et le bien-être de l'âme (inculquer une croyance vraie, celle en l'existence et l'unicité de Dieu par exemple) $)^{33}$. Le recours à une argumentation de type historique s'impose à Maïmonide essentiellement lorsqu'il s'agit de rendre compte de l'utilité des lois relatives à l'interdit de l'idolâtrie ou plus précisément lorsqu'il s'agit de les expliquer en détail. Car dans leur principe, toutes les lois relatives à l'interdit de l'idolâtrie s'inscrivent totalement dans la définition d'une loi divine, puisqu'elles visent à inculquer des vérités spéculatives, l'unicité de Dieu et son immatérialité, et à amener au bien-être corporel en libérant le peuple « de toutes ces 
pratiques pénibles $\aleph^{34}$, que constituaient, selon Maïmonide, les lourdes charges religieuses imposées par l'idolâtrie. Reste à en expliquer le détail et à justifier la conservation de certaines pratiques, comme celle des sacrifices, qui entre apparemment en contradiction avec l'affirmation maïmonidienne qu'il suffit, pour servir Dieu, de « l'aimer » et de le « craindre " ${ }^{35}$.

Le célèbre chapitre III, 32 du Guide, expose les raisons du maintien des rites sacrificiels dans la Torah. L'argument est déjà historique dans son principe. Il suit une forme strictement syllogistique : la prémisse majeure est l'affirmation anthropologique que «l'homme, selon sa nature, ne saurait quitter brusquement toutes ses habitudes $»^{36}$; la mineure dresse le constat historique qu'au moment du don de la Torah «c'était une coutume répandue, familière, au monde entier - et nous-mêmes avions été élevés dans ce culte universel - d'offrir diverses espèces d'animaux dans ces temples où l'on plaçait les idoles $\aleph^{37}$. Il en résulte qu'il eût été impossible de faire accepter la Torah aux Hébreux si elle avait imposé l'abandon total et immédiat du culte sacrificiel. C'est pourquoi la Torah maintient la pratique des sacrifices tout en s'efforçant de la restreindre, interdisant toute offrande en dehors du Temple et encadrant leur pratique par des règles très strictes. En d'autres termes, pour reprendre l'expression d'Amos Funkenstein, Dieu s'accommode de la nature humaine ${ }^{38}$. Loin de s'imposer par l'évidence de sa révélation aux Hébreux, ainsi que le rapporte le Mishneh Torah, la Torah doit négocier dans le Guide avec leurs habitudes pour être acceptée par eux.

Lorsqu'il s'agit de rendre compte des lois sacrificielles de la Torah dans leurs détails, la distance qui sépare ce que l'on trouve dans chacune des deux œuvres s'accroît. Rambam s'efforce en effet dans le Guide de fournir les raisons qui motivent l'ensemble des commandements de la Torah, dans leurs dispositions générales et, dans la mesure du possible, particulières ${ }^{39}$, pour démontrer au lecteur "perplexe » leur utilité ainsi que la compatibilité du « sens extérieur » de la Loi avec la raison. Or, en ce qui concerne les sacrifices, l'explication de leurs dispositions de détail requiert, apparemment du moins, la transgression d'un interdit halakhique, rapporté bien évidemment dans le Mishneh Torah : celui de s'enquérir des pratiques des idolâtres et, pire encore, de les exposer par écrit.

Ainsi, peut-on lire au chapitre 2 des Hilkhot 'avodah zarah (Règles relatives à l'idolâtrie) :

Les idolâtres ont composé de nombreux livres sur leur culte et y ont expliqué quel en est le principe, quels en sont les actions et les jugements qu'il commande. Le Saint, béni-soit-il, nous a prescrit de ne rien lire de ce qui se trouve dans ces livres et de n'attacher notre pensée ni à l'idolâtrie, ni à rien de ce qui y ressortit ${ }^{40}$.

La halakhah suivante ainsi que le Livre des commandements (Sefer ha-miswot) ${ }^{41}$ précisent en outre que celui qui enfreint cet interdit encourt la flagellation ${ }^{42}$. Or, nous lisons dans le Guide :

Je dis que ce qui m'a fait comprendre le sens d'un grand nombre de commandements et ce qui m'en a fait connaitre la raison, c'est l'étude que j'ai faite des doctrines des Sabiens [les idolâtres], de leurs opinions, de leurs pratiques et des cérémonies de leurs cultes ${ }^{43}$.

Maïmonide va jusqu'à inviter son lecteur à lire certains des livres, alors disponibles en arabe, décrivant ces pratiques et à en donner un long résumé ${ }^{44}$. Seule la connaissance historique des pratiques des idolâtres à une époque supposée contemporaine du don de la Torah permet, selon lui, de comprendre certaines dispositions de la Loi visant à abroger l'idolâtrie. Ainsi, Maïmonide explique-t-il par exemple le fait que la Torah prescrive de sacrifier précisément des boucs, des béliers et des bœufs par le fait que ces trois espèces étaient un objet de culte chez la plupart des idolâtres. De même, 
l'interdiction d'accompagner les sacrifices de miel ou de levain ainsi que la prescription de les offrir avec du sel sont expliquées comme étant à l'opposé de la plupart des cultes idolâtriques contemporains de l'époque de Moïse ${ }^{45}$. Ce n'est donc qu'au prix de ce qui paraît, au premier abord, être une grave transgression de la Loi que Maïmonide peut exposer à son destinataire "perplexe » les motivations rationnelles de commandements apparemment arbitraires et en dernière instance, le caractère divin de la Torah.

Mais c'est précisément parce qu'il s'adresse à un tel destinataire, que Maïmonide se permet une telle transgression. Dans le Livre des commandements, Maïmonide commente l'interdit de s'intéresser aux cultes idolâtres, en l'expliquant par le danger de se trouver séduit, comme envoûté par eux. L'idolâtrie exerce, semble-t-il, un pouvoir de fascination quasi irrésistible. Néanmoins, à regarder le texte de Maïmonide de plus près, il apparaît que ce n'est pas le culte idolâtrique en tant que tel qui est pourvu d'un tel pouvoir d'envoûtement; c'est au contraire une certaine manière de s'y intéresser qui risque de conduire à une erreur concernant le culte en général. Maïmonide souligne en effet subtilement que ce que proscrit la Torah est à strictement parler une attitude de curiosité vis-à-vis des cultes idolâtres :

Ainsi il est dit De peur que tu investigues sur leurs dieux en disant : « Comment ces peuples servent-ils leurs dieux ? Et j'en ferai de même, moi aussi » (Dt 12, 30), pour interdire de s'interroger sur leur manière de rendre le culte, $y$ compris sans rendre ce culte soi-même $e^{46}$.

$\mathrm{Du}$ style interrogatif du verset, on déduit que ce qui se trouve interdit n'est pas seulement le culte en tant que tel, mais avant tout une approche strictement culturelle du culte étranger. S'intéresser aux pratiques de l'autre par simple curiosité ou par simple tempérament cosmopolite, c'est prendre le risque d'oublier que le culte n'a pas qu'un intérêt documentaire, mais a fondamentalement pour enjeu un rapport au divin, un rapport au monde et un rapport à soi.

On voit par ailleurs que l'interdit de s'intéresser aux cultes idolâtriques n'est pas un interdit absolu, mais qu'il y existe une exception. Parce que la pratique de l'idolâtrie se traduit, en principe, par une peine prononcée par un tribunal rabbinique, c'est une obligation pour les juges de connaître les pratiques des idolâtres ${ }^{47}$. Les juges dès lors peuvent et même doivent se renseigner sur l'idolâtrie, car leur démarche, n'étant pas alors motivée par la seule curiosité, mais par l'application de la Torah, ne les expose pas au risque de la séduction qui leur ferait oublier que le cultuel n'est pas réductible au culturel.

C'est néanmoins pour une autre raison que Maïmonide s'autorise, dans le Guide, à enfreindre l'interdit de se renseigner sur l'idolâtrie. Il ne s'agit pas, en l'occurrence, de le faire pour appliquer la Torah, mais pour la fonder comme loi divine. On peut prendre le risque d'exposer ces pratiques au destinataire philosophe du Guide, parce que sa démarche également n'est pas documentaire ou curieuse, elle est exigeante et relève de l'interrogation sur les fondements de sa croyance. Il exige que le caractère divin de la Loi, auquel il ne fait que croire du fait de son éducation, lui soit démontré rationnellement. Et Maïmonide ne peut répondre à cette exigence qu'à la condition de transgresser quelques dispositions de la Loi, se plaçant sous l'autorité du verset « C'est le moment d'agir pour Dieu; [on a violé ta Torah.] » (Ps 119, 126), traditionnellement interprété comme autorisant de transgresser la Loi, s'il s'agit de la renforcer ou de la 
fonder ${ }^{48}$. L'interdit de se documenter sur l'idolâtre est ainsi devenu, dans cette démarche fondationnelle, obligation de le faire.

La comparaison du traitement du caractère divin de la Torah dans le Mishneh Torah et dans le Guide permet, en tout cas, de bien saisir la différence de perspectives qui sépare les deux ouvrages. Au début de la vaste entreprise de compilation de la loi orale que constitue le Mishneh Torah, Maïmonide se doit d'exposer en détail les fondements de la Torah. Assurément, la croyance en la prophétie et en la «métaprophétie » de Moïse, autrement dit, au caractère révélé de la Torah, a sa place parmi ces fondements. Elle n'a pas à être démontrée à un lecteur qui se soucie de savoir comment agir conformément à la Loi. Dans le Guide, Maïmonide ne peut se contenter d'exposer ces fondements : il les interroge et s'attache à les prouver. Pour reprendre les termes de la parabole qui figure dans l'un des derniers chapitres du Guide (III, 51), le destinataire du Mishneh Torah fait partie (au mieux) de ceux qui sont arrivés jusqu'au palais et qui tournent autour sans en trouver l'entrée, « les casuistes qui admettent, par tradition, les opinions vraies, qui discutent sur les pratiques du culte, mais qui ne s'engagent point dans la spéculation sur les principes fondamentaux de la religion, ni ne cherchent en aucune façon à établir la vérité d'une croyance quelconque $»^{49}$; celui du Guide est quant à lui entré dans le vestibule, faisant partie de « ceux qui se plongent dans la spéculation sur les principes fondamentaux de la religion $»^{50}$.

Il est donc tentant d'opposer une supposée doctrine (ésotérique) de la Loi spécifique au Guide à une supposée doctrine (exotérique) de la Loi propre au Mishneh Torah, à l'instar d'une certaine tradition universitaire après Léo Strauss ${ }^{51}$. Néanmoins, si on abandonne la question des fondements de la Loi, pour s'interroger sur celle de son statut, on découvre sur ce point une doctrine rigoureusement unitaire dans l'œuvre de Maïmonide. En effet, si le Guide renonce à présupposer le caractère divin de la Torah, il n'affirme pas moins, après avoir prouvé son caractère divin, sa perpétuité et l'interdiction d'y rien ajouter ou retrancher ${ }^{52}$. Paradoxalement, si la preuve de l'origine divine de la Torah nécessitait le recours à l'argument historique, cette origine divine une fois prouvée impose que la Torah est parfaite et qu'elle ne saurait être modifiée. Inversement, dans le Mishneh Torah, on rencontre également des occurrences de l'argument historique, notamment pour expliquer les lois relatives à l'interdit de l'idolâtrie (par exemple, l'interdiction de se raser les cinq «bords» du visage) ${ }^{53}$. Maïmonide ne recule pas devant cette contradiction apparente et c'est précisément dans son caractère à la fois historiquement daté et universellement valable qu'il fait résider l'exceptionnalité de cette Loi.

\section{La question du statut de la Loi}

Les deux propositions principielles de cette doctrine maïmonidienne de la Loi sont les suivantes : 1/ la Torah n'est pas une fin en soi, elle n'est qu'un moyen en vue d'une fin qui lui est extérieure - à savoir l'avènement de la perfection humaine, ce qui en termes aristotéliciens s'énonce comme étant l'accès à la vie contemplative de la partie rationnelle de l'âme; 2/ pour autant, l'observance de ses commandements est absolument nécessaire à cette fin. Cette double thèse s'exprime bien évidemment de manière très différente dans le Mishneh Torah et dans le Guide du fait de la différence de leurs destinataires et de leur destination, mais elle est bien présente dans l'une et l'autre œuvre. 


\section{La vie du monde à venir dans le Mishneh Torah}

Dans le Mishneh Torah, on la rencontre dans le cadre de la doctrine de la rétribution. Cette doctrine s'inscrit dans le cadre des Hilkhot teshuvah (règles relatives à la repentance, litt. du retour), que Maïmonide comprend comme un véritable mouvement de rapprochement de l'homme vers $\operatorname{Dieu}^{54}$. Ce n'est sans doute pas anodin: ce rapprochement constitue déjà par lui-même une forme de rétribution ${ }^{55}$. Car dans cette section qui figure à la fin du Sefer ha-maddac, l'auteur affirme que la véritable récompense que l'homme acquiert par le respect des commandements est ce que la littérature rabbinique désigne comme étant la vie du " monde à venir " ('olam ha-ba'). ${ }^{56}$ Maïmonide comprend cette "vie» comme étant la vie purement contemplative de "l'âme rationnelle" après la mort. La partie rationnelle de l'âme humaine qui constitue la faculté d'accéder à la connaissance des intelligibles, réalités indépendantes de l'existence matérielle, est ce que désigne le verset « Faisons l'homme à notre image, à notre ressemblance (be-șalmenu ki-dmutenu). » (Gn 1, 26), selon l'interprétation qu'en donne Maïmonide au début du Mishneh Torah comme du Guide. ${ }^{57}$ Ainsi, après la mort,
... lorsque se désagrège la matière composée des éléments, le souffle vital périt également, car
il n'a pas d'existence indépendante du corps de sorte que toute son activité en dépend. Mais
la forme de l'âme [i.e. sa partie intellective] n'est pas anéantie, car son activité ne dépend
pas du souffle vital. Elle saisit et appréhende les connaissances indépendantes de la matière
[i.e. les intelligibles]. Elle connaît aussi le Créateur de toutes choses et subsiste à jamais
dans les siècles des siècles ${ }^{58}$.

Telle est donc l'ultime récompense de l'homme observant : l'accès à cette vie éternelle de l'âme déliée de la matière et consacrée à la contemplation permanente de Dieu. Le plus haut degré de punition de l'homme mauvais est au contraire l'exclusion de la vie du monde à venir, l'absence de toute compréhension de l'essence de Dieu, la peine dite de « retranchement» (karet). L'observance des lois de la Torah en ce monde ('olam ha$z e h)$ n'est donc qu'une préparation à la vie du monde à venir. C'est ce que laisse du moins entendre la conception maïmonidienne des temps messianiques, selon laquelle ceux-ci se réduisent à la libération du peuple d'Israël du joug des nations, à la restauration de la royauté en Terre Sainte et, avec elle, à l'accomplissement de tous les commandements de la Torah (y compris le service du Temple). Maïmonide y insiste : les temps messianiques ne verront en rien une transformation ou une transfiguration de l'ordre $\mathrm{du}$ monde $\mathrm{e}^{59}$. Ils ne sont rien d'autre qu'une époque historique au cours de laquelle, d'une part, tous les peuples reconnaîtront Dieu et s'attacheront à le servir (en appliquant les sept lois qui leur furent prescrites par Dieu, dites « lois noachiques ») et, d'autre part, il sera possible à Israël d'appliquer les commandements (et tous les commandements) de la Torah et, par conséquent, de bénéficier d'un accès plus aisé à la vie du monde à venir ${ }^{60}$ :

\footnotetext{
Et telle est la raison pour laquelle tous les enfants d'Israël, leurs prophètes et leurs sages, ont désiré ardemment l'avènement des temps du roi-messie: afin qu'ils soient libérés de l'oppression politique qui ne les laisse pas s'appliquer en paix à l'étude de la Torah et à l'application de ses commandements comme il convient - et la quiétude dans laquelle ils se trouveront alors leur permettra de croitre en sagesse afin d'obtenir la vie du monde à venir. C'est parce que les temps messianiques verront croître connaissance, sagesse et véritéb.
} 


\section{Le « culte véritable » du Guide}

La langue du Mishneh Torah, s'exprimant en termes de "récompense» ou de «rétribution» (littéralement "salaire», sakhar), laisse à penser que la vie contemplative de l'âme n'est qu'une faveur, un octroi divin, qu'elle se joue dans une dimension totalement autre. Maïmonide insiste d'ailleurs sur "l'incommensurabilité " du monde à venir ${ }^{62}$. Dans ce monde-ci du moins, l'observance des commandements peut encore apparaître comme une fin en soi. Le Guide abandonne quant à lui le vocable pédagogique ${ }^{63} \mathrm{de}$ la " récompense » et la référence au monde à venir pour analyser plus en détail en quoi la Loi peut constituer une préparation nécessaire à la vie contemplative. L'accès aux vérités spéculatives n'y semble pas totalement impossible à l'homme avant sa mort. Il n'en reste pas moins extrêmement difficile et suppose la capacité, exceptionnelle pour un être humain, de s'abstraire du souci des choses sensibles pour exercer son âme à la connaissance des intelligibles et en dernière instance, dans la mesure du possible, de Dieu. Cette activité méditative est présentée à maintes reprises dans le Guide, et en particulier au chapitre III, 51 déjà cité, comme le « vrai culte $»^{64}$, le « culte du cœur $»^{65}$, le « culte intellectuel $»^{66}$ ou encore le « grand et véritable culte $\aleph^{67}$, laissant entendre que ce culte authentique est à opposer à un culte de moindre valeur, sinon sans valeur aucune : la pratique des commandements de la Torah en tant que telle, dépourvue de contemplation.

Mais immédiatement après avoir fait l'apologie de cette religion spirituelle et méditative, Maïmonide insiste sur l'insuffisance de ce culte : il est inséparable de la pratique de la Loi. Celle-ci est en effet, nous l'avons vu, la loi parfaite, loi par conséquent sans aucun équivalent. En améliorant le bien-être du corps (par ses prescriptions politiques) et le bien-être de l'âme (par les idées relevant de la physique et de la métaphysique qu'elle enseigne), elle libère l'homme de ses préoccupations mondaines et l'aide à concentrer son âme sur les vérités spéculatives ${ }^{68}$. Elle constitue ainsi une étape préparatoire et nécessaire à l'accès au « culte intellectuel ». Au dernier chapitre du Guide (III, 54), décrivant les quatre types de perfections accessibles à $l^{\prime}$ homme (par ordre d'importance, la perfection : $1^{\circ}$ dans les possessions, $2^{\circ}$ physique, $3^{\circ}$ morale, $4^{\circ}$ intellectuelle), Maïmonide décrit les pratiques de la Loi comme le moyen de parvenir à la perfection morale, qui n'est elle-même qu'une préparation à la perfection intellectuelle ${ }^{69}$ Le caractère nécessaire de cette préparation est par ailleurs explicité par une gradation de la crainte et de l'amour de Dieu:

... c'est en se livrant à tous ces détails pratiques et en les répétant, que certains hommes

d'élite s'exerceront pour arriver à la perfection humaine, de manière à craindre Dieu, à le respecter et à le révérer...

et, plus loin :

En effet, ces deux buts, à savoir l'amour et la crainte de Dieu, sont atteints par deux choses:

à l'amour on arrive par les idées que renferme la Loi sur la doctrine véritable de l'existence de Dieu; à la crainte on arrive au moyen de toutes les pratiques de la Loi, comme nous l'avons exposép.

La pratique des commandements seule conduit à la crainte et au respect de Dieu, en lui soumettant la volonté des hommes; elle permet à l'homme de tourner son âme vers la connaissance de Dieu, ce qui en constitue le véritable amour. Le Mishneh Torah donnait déjà une interprétation du commandement prescrivant "d'aimer Dieu », qui figure au chapitre des fondements de la Torah (yesodei ha-Torah), en termes de connaissance de Dieu et de reconnaissance de Dieu comme Créateur du monde ${ }^{72}$. Telle est donc la place 
assignée à la Loi aussi bien dans le Guide que dans le Mishneh Torah : elle vise à amener à la crainte de Dieu qui seule permet l'amour de Dieu, qui anticipe elle-même sur ce que le Mishneh Torah appelle « vie du monde à venir » et le Guide « culte intellectuel ».

Le Guide précise, en outre, qu'il revient aux «hommes d'élite ", qui ont eux-mêmes besoin de passer par la pratique de la Loi, d'enseigner la Loi au peuple et de l'inciter à l'appliquer, dans la lignée des patriarches qui avaient pour but principal de «faire naître une nation qui connaîtrait Dieu et l'adorerait $" .^{73}$ Ainsi l'itinéraire du sage authentique est-il de s'enquérir d'abord du sens de la Loi, après l'avoir pratiquée, puis de parvenir à la science philosophique, la connaissance métaphysique et physique, pour enfin veiller à la bonne application de la Loi au sein du peuple, de manière à ce que puisse naître en son sein d'autres hommes sages et justes. Dans la parabole de la fin du Guide, celui qui est parvenu jusque dans la salle où trône le seigneur du palais se doit d'assurer la perpétuité de sa Loi :

Les docteurs disent encore qu'on exige de l'homme d'abord la science de la Loi, ensuite la science (philosophique), et enfin la connaissance de la tradition qui se rattache à la Loi, c'est$\grave{a}$-dire de savoir en tirer des règles pour sa conduite. Tel doit être l'ordre successif des études: d'abord on doit connaître les idées en question traditionnellement, en suite on doit savoir les démontrer, et enfin on doit se rendre un compte exact des actions qui constituent une bonne conduite ${ }^{74}$.

Reste à comprendre comment cette Loi, dont certaines dispositions ont été expliquées, dans le Guide, comme inscrites dans un contexte historique précis, peut valoir en tout temps comme préparation à la perfection intellectuelle de l'homme.

\section{L'idolâtrie comme concept}

Il faut pour cela se pencher sur la signification de l'interdit de l'idolâtrie, qui est le principal point, nous l'avons vu, à propos duquel Maïmonide avance une explication de type historique. Dans le Sefer ha-madda', Maïmonide se penche sur les origines de l'idolâtrie. Elle apparaît comme une perversion du monothéisme originel des hommes. Les hommes de la génération d'Énoch, petit-fils d'Adam, commencèrent à rendre un culte aux astres, pensant honorer le Créateur par l'intermédiaire de ses créatures les plus éminentes. De génération en génération, ce culte devint de plus en plus important et imposant, si bien qu'on finit par oublier qu'il était avant tout adressé à Dieu. ${ }^{75}$ Telle est la logique perverse de l'idolâtrie : ériger l'intermédiaire en obstacle, d'où la manière maïmonidienne de jeu autour de l'homonymie des verbes סרסר (sarser, servir d'intermédiaire) et סור (sor, se détourner) :

[...] que le désir de votre cour ne vous trompe pas en vous poussant à servir ces créatures

[les astres] et à établir un intermédiaire (sarsur) entre le Créateur et vous ${ }^{76}$.

L'erreur des idolâtres n'est donc pas principalement de penser que les statues qu'ils ont eux-mêmes fabriquées sont des divinités. Cette croyance abjecte n'est que la conséquence d'une erreur bien plus fondamentale, qui consiste simplement à détacher le culte de son véritable objet. Autrement dit, l'essence de l'idolâtrie consiste à penser que le culte a une valeur en lui-même, indépendante de celui auquel il s'adresse, voire qu'il n'est pas même adressé, comme le précise un passage de la première partie du Guide :

... ces mécréants, tout en croyant l'existence de Dieu, appliquaient leur fausse croyance à quelque chose qui n'est dû qu'à Dieu seul, [...] et croyaient pouvoir rendre ce devoir à ce qui est hors de lui (chose qui contribuait à faire disparaître l'existence de Dieu de la croyance du 
peuple, celui-ci ne saisissant que les pratiques du culte sans en pénétrer le sens, ni connaître

la réalité de celui à qui s'adresse ce culte)..." ${ }^{77}$

Ainsi, un culte est idolâtre, même s'il vise à servir à Dieu, lorsqu'il s'érige entre celui qui sert et celui qu'il pense servir. Idolâtrique qualifie l'autonomisation du culte, en tant que pur rite, pure pratique, sans adresse donc sans signification. L'affirmation maintes fois répétée dans le Guide que la Loi a pour but principal la destruction de l'idolâtrie ${ }^{78}$ signifie donc que la Torah a été donnée essentiellement pour combattre l'idée d'une loi valable pour elle-même sans référent extérieur, transcendant. À ce titre, par parenthèse, il faut dire en suivant Maïmonide que considérer l'application des commandements de la Torah comme une fin en soi est une forme particulièrement perverse de l'idolâtrie ${ }^{79}$ ! On veut parler ici d'une orthopraxie aveugle, qui ne s'interroge ni sur sa raison d'être, ni sur son adresse, qui fonctionne mécaniquement ou par simple inertie sociale. L'idolâtrie, en tous cas, de la simple perversion du monothéisme qu'elle était à l'origine, est devenue avec le temps sa pure et simple négation en tant que négation de toute transcendance au monde.

De plus, si l'explication donnée dans le Guide de certaines lois relatives à l'interdit de l'idolâtrie consiste en l'explicitation du contexte historique de leur énonciation, la séduction exercée par l'idolâtrie est présentée dans l'ensemble de l'œuvre maïmonidienne comme constituant un danger permanent et universel. Comme nous l'avons déjà mentionné, dans le Mishneh Torah comme dans le Livre des commandements ${ }^{80}$, l'interdit de porter son intérêt sur les livres et les pratiques des idolâtres est justifié précisément par ce danger de séduction. Dans le Guide également, Maïmonide met en garde son lecteur de façon tout à fait étonnante contre la fascination que peuvent exercer les écrits des idolâtres :

... il faut te bien garder de te laisser troubler l'esprit et de t'imaginer que ce qu'ils [les Sabiens] disent soit jamais arrivé à Adam ou à un autre. [...] Bien qu'un homme comme toi n'ait pas besoin de cette observation [...], j'ai pourtant cru devoir donner un avertissement pour préserver les autres; car le vulgaire n'est que trop disposé à ajouter foi aux fables ${ }^{81}$.

Nous avons précédemment exclu que le destinataire du Guide soit concerné par le risque de séduction d'une approche purement documentaire du culte. Comment comprendre alors que le seul fait de prendre connaissance des cultes des anciens idolâtres, depuis fort longtemps disparus à l'époque où Maïmonide écrit le Guide, apparaisse ici comme exposant l'homme, en général, et y compris le philosophe, au danger de leur fascination? Cette question s'impose d'autant plus que, dans le même chapitre, Maïmonide affirme la persistance, en son temps, de certaines formes d'idolâtrie :

Si la croyance à l'existence de Dieu n'était pas si généralement reconnue dans les religions actuelles, il y aurait de nos jours des ténèbres plus épaisses encore que celles qui régnaient en ces temps-là ; cependant il y en a à d'autres égards ${ }^{82}$.

Peu importe si Maïmonide vise ici les croyances superstitieuses très répandues en son temps, comme l'indique Salomon Munk dans une note sur ce passage. «Il y en a à d'autres égards" signifie que l'idolâtrie bien qu'elle ait abandonné depuis fort longtemps sa forme originelle subsiste au cours de l'histoire en revêtant d'autres formes. Ce n'est donc pas l'idolâtrie dans la forme historique qu'elle avait au moment supposé du don de la Torah qui est ici visée, mais bien l'idolâtrie comme concept, telle que nous l'avons dégagée plus haut, comme tendance générale à se satisfaire de ce qui est, tel qu'il est, en en oubliant d'en interroger le sens et l'origine, qui, elle, menace 
perpétuellement et sous toutes ses formes, archaïques comme modernes, que ce soit celle de croyances superstitieuses ou du $"$ fétichisme de la marchandise ${ }^{83}$.

Ainsi, que la Loi, qui constitue l'objet d'une révélation historique, proscrive l'idolâtrie sous une forme elle-même historique, ne rend pas pour autant cette Loi caduque en des temps plus "avancés » où l'idolâtrie a disparu sous cette forme précise, puisque l'idolâtrie en tant que telle y subsiste.

\section{La Torah : loi à étudier en totalité, mais applicable seulement en partie}

Deux distinctions établies par Maïmonide, l'une dans le Guide, l'autre dans le Livre des commandements, permettent de mieux saisir ce point. La première est la distinction entre dispositions générales et dispositions de détail des commandements. Au chapitre III, 26, du Guide, Maïmonide applique cette distinction aux commandements relatifs aux sacrifices, indiquant que les sacrifices en général ont une raison évidente - éloigner le peuple juif de l'idolâtrie -, mais que le détail de ces commandements est la plupart du temps inexplicable :

... si tu demandais, par exemple, pourquoi (on devait offrir) un agneau et non pas un bélier, on pourrait faire exactement la même question si on avait dit bélier au lieu d'agneau, puisqu'il fallait une espèce quelconque. [...] Cela ressemble en quelque sorte à la nature du possible, où il faut nécessairement qu'il arrive une d'entre les choses possibles, sans qu'on ait le droit de demander pourquoi telle d'entre ces choses a eu lieu et non pas telle autre d'entre les choses possibles; car on pourrait faire la même question si une autre chose possible s'était réalisée au lieu de celle-là ${ }^{84}$.

On voit ici encore à l'œuvre l'idée d'accommodation. Pour interdire l'idolâtrie, il fallait que Dieu, s'accommodant de la nature humaine, prescrive des lois sacrificielles. Et pour prescrire de telles lois, il fallait qu'elles incluent des dispositions de détails, ellesmêmes contingentes ${ }^{85}$. Cette idée fonctionne également à un échelon supérieur, au niveau de l'interdiction de l'idolâtrie elle-même. Il fallait que l'interdiction de l'idolâtrie prît une forme ou une autre, cela relève comme le dit Maïmonide « de la nature du possible »; elle a pris celle des sacrifices parce que la Torah a été donnée à un moment où ils incarnaient l'idée d'idolâtrie, sous-entendue, elle aurait pu prendre une autre forme. Mais cela n'a aucune espèce d'incidence sur l'édifice et la destination générale de la Loi.

La seconde distinction éclairante sur la question est celle que Maïmonide établit, dans le Livre des commandements, dans son exposé du $187^{\mathrm{e}}$ commandement positif, prescrivant l'extermination des peuples cananéens (peuples qui, dans la Torah, constituent l'incarnation même de l'idolâtrie) :

De manière générale, il faut comprendre et faire la différence entre un commandement et ce à quoi il se rapporte. En effet, il existe des commandements applicables à toute époque, mais dont l'objet [nécessaire à leur exécution] peut faire défaut à une époque donnée. Toutefois, ce n'est pas en raison de la disparition de l'objet de ce commandement que celui-ci deviendra non applicable en tout temps (Tibbon: noheget le-dorot) ${ }^{86}$.

En d'autres termes, qu'une loi ne soit pas applicable du fait de l'absence de son objet à une époque déterminée n'empêche pas qu'elle soit valide à toute époque. En l'occurrence, que les peuples cananéens de la Torah n'existent plus à l'époque de Maïmonide n'invalide pas pour autant le commandement de les exterminer. Mais cela est également vrai des lois qui nécessitent l'existence d'un Temple ou la royauté 
d'Israël : quoique inapplicables depuis la destruction du second Temple, elles n'en restent pas moins valides. Pour revenir à l'exemple de l'interdit de l'idolâtrie, toutes les lois qui s'inscrivent dans son cadre sont valides en tout temps, même si certaines d'entre elles, en particulier les lois sacrificielles, sont inapplicables au temps de Maïmonide comme aujourd'hui.

Maïmonide fait ainsi de la Torah une loi éternellement valable quoiqu'en grande partie inapplicable. Il revient aux Juifs de connaître la Loi dans sa totalité, bien qu'il leur soit impossible, à l'époque de Maïmonide comme aujourd'hui, d'en appliquer toutes les dispositions. La Loi devient ainsi objet d'étude et de méditation et non de fascination, en quoi elle réussit dans sa lutte contre l'idolâtrie. Ainsi, la description par Maïmonide, dans le Guide, d'une religion en progrès, qui passerait du "stade" sacrificiel au « stade » de la pure méditation, en passant par celui du remplacement des sacrifices par la prière ${ }^{87}$, n'est pas aussi incompatible qu'il y paraît au premier abord avec sa conception restauratrice du messianisme, dont voici l'énoncé le plus cinglant :

Le roi-messie viendra, restaurera le royaume de David dans sa puissance première, rebâtira le Sanctuaire et rassemblera les dispersés d'Israël. Toutes les lois seront rétablies à cette époque, comme elles étaient dans les temps antiques. Les sacrifices seront de nouveau offerts; l'année sabbatique et le jubilé seront observés exactement suivant les prescriptions de la Torah. Celui qui ne croit pas au roi-messie ou qui n'attend pas sa venue rejette non seulement les autres prophètes, mais aussi la Torah et notre maître Moïse ${ }^{88}$.

Les temps messianiques ne sont rien d'autre que la Torah en acte, appliquée dans toute son extension. Dès lors, cette version radicalement déflationniste du messianisme, en regard de ses courants utopistes et apocalyptiques ${ }^{89}$, établit un rapport direct entre le rejet de l'espérance messianique et le rejet de la Torah comme telle. En donnant à espérer un monde totalement renouvelé dans les temps messianiques, les tendances utopiques et apocalyptiques contiennent en germe ce que Scholem nomme «l'antinomisme» de certains mouvements messianiques (en particulier le sabbataïsme), autrement dit une remise en cause du caractère éternel de la Torah ${ }^{90}$. La croyance en des temps messianiques de stricte restauration proposée par Maïmonide garantit au contraire la validité universelle de la Loi. Certes, Maïmonide fait, dans l'Épître au Yémen ${ }^{91}$, un usage strictement politique des ressources inhérentes à la promesse messianique. Contre ses propres principes, il va jusqu'à avancer une date de la venue prochaine du messie, dans le but de rassurer la communauté juive du Yémen en proie à de violentes persécutions et à la tentation de l'apostasie. Mais on ne saurait réduire l'interprétation maïmonidienne du messianisme à une manœuvre politique. En affirmant que la Torah dans son ensemble sera restaurée dans les temps messianiques, Maïmonide soutient paradoxalement que, quoique largement inapplicable aujourd'hui comme en son temps, elle nous concerne en chacune de ses parties ${ }^{92}$. Attendre ardemment la venue du messie revient, dans cette perspective purement restauratrice, à exprimer le désir de la perpétuité de cette Loi.

Cette Loi dont une des prescriptions principales est de s'occuper constamment à son étude, quand bien même elle ne saurait être totalement appliquée, semble propre, dans l'édifice maïmonidien, à jouer en tout temps le rôle d'une préparation au «culte véritable » que constitue l'application de l'intellect à la connaissance des intelligibles et, ultimement, de Dieu.

Ainsi, s'il est une contradiction entre le Mishneh Torah (plus particulièrement, le Sefer ha-madd $a^{a}$ ) et le Guide des égarés eu égard à la question de la Loi, elle est à chercher du côté des fondements de cette Loi. Le Mishneh Torah postule le caractère révélé, parfait et 
éternel de la Torah, à travers une interprétation généreuse de la scène du Sinaï. Le Guide s'efforce quant à lui de l'établir, de manière à transformer la simple croyance en la vérité de la Loi, remise en cause, chez son destinataire «perplexe ", par la rencontre de la philosophie, en une certitude rationnellement fondée. C'est, du fait de cette différence de perspective et d'adresse qu'apparaît l'apparente antinomie entre ce que nous avons appelé l'argument "historique» et l'affirmation de l'immutabilité de la Loi. Cette contradiction peut être levée, à condition d'abandonner la question des fondements de la Loi pour se pencher sur celle de son statut. La Loi n'est qu'un moyen - mais le seul dont nous disposons - en vue de l'accès à la vie contemplative de l'âme intellective. Elle vise à assurer le bien-être du corps (politique), qui seul garantit à l'homme la capacité de se consacrer à l'exercice de la pensée, et le bien-être de l'âme en inculquant les bases des vérités spéculatives. Elle peut assurer une telle fonction de manière universelle, malgré le caractère "historique» de certaines de ses parties, parce que ces parties " historiques » sont précisément celles qui ne sont pas applicables en tout temps, en l'absence d'un Temple, quoique universellement valides. Il est donc une obligation pour les Juifs de prendre connaissance de ces lois, de les étudier du moins, alors qu'il leur est interdit, à l'heure actuelle, comme au temps de Maïmonide, de les appliquer. À ce titre la contradiction entre l'argument historique et le principe de l'immutabilité de la Loi peut être dépassée.

En donnant à la Torah le statut d'une Loi universelle, en large partie suspendue dans son application, Maïmonide fournit certes une puissante défense d'un judaïsme affaibli par les persécutions, en proie au doute, aux dissensions et à la dispersion. Cette défense ne se réduit pourtant pas à un souci maïmonidien de préserver sa communauté «religieuse » ou «nationale» de la crise politique qu'elle endure; elle consiste au contraire à assurer au judaïsme et à sa Loi l'universalité d'un concept : celui de lutte contre l'idolâtrie, entendue comme soumission aveugle à des normes, dont ni le sens ni l'origine ne sont jamais interrogés.

\section{NOTES}

1. Shlomo Pinès, "Quelques réflexions sur Maïmonide en guise de préface ", in Le Livre de la connaissance, trad. Valentin Nikiprowetzky et André Zaoui, Paris, PUF, 1961, p. 17.

2. Maïmonide, Guide des égarés. Traité de théologie et de philosophie, trad. Salomon Munk, Paris, Maisonneuve \& Larose, 2003 [1866], désormais noté Guide.

3. Mishneh Torah. Hu' ha-yad ha- hazaqah le-ha-Rambam, Jérusalem, Shabetay Fraenkel, 1989. Pour les citations du Sefer ha-madda', nous renvoyons à la traduction française, en la modifiant au besoin.

4. Cf. "Introduction au chapitre Hélèq ", in. Épitres, trad. [de l'hébreu] Jean de Hulster, Paris, Verdier, 1983, p. 185-192. (Pour une traduction hébraïque, cf. Haqdamot ha-Rambam, Yi șhaq Shailat (éd.), Jérusalem, Ma'aleh Adumim, 1997.)

5. Le Livre de la connaissance, op. cit., p. 87 (traduction modifiée). Hilkhot yesodei ha-Torah, 7, 5.

6. Ibid., p. 92-93. Hilkhot yesodei ha-Torah, 8, 1. 
7. Recueil de textes lus les deux premiers soirs de Pesaḥ (en Terre Sainte, le premier soir seulement), contenant notamment le récit de la sortie d'Égypte.

8. «Ainsi, tout Israël est, après la scène du mont Sinaï, autant de témoins pour [la prophétie de] Moïse et il n'est donc pas besoin de produire des signes [pour qu'ils y croient].» (Ibid., p. 93. Hilkhot yesodei ha-Torah, 8, 2.)

9. Ibid., p. 95. Hilkhot yesodei ha-Torah, 8, 3. L'argument complet est le suivant : c'est parce que la Torah de Moïse nous enjoint à croire aux signes et aux prodiges des prophètes que nous devons les croire. Par conséquent, une prophétie qui abrogerait la loi de Moïse est proprement contradictoire puisqu'elle annule les conditions mêmes de sa réception.

10. Maïmonide interprète ici les miracles accomplis par Moïse (l'ouverture de la mer Rouge et la submersion des Égyptiens, la manne, l'extermination de la bande de Coré) non comme des preuves de sa prophétie, mais comme des réponses à la nécessité du moment (cf. Ibid., p. 92. Hilkhot yesodei ha-Torah, 8, 1).

11. Il s'agit, dans la typologie de la prophétie que propose Hannah Kasher, dans un article récent, du cas du prophète $1 /$ qui se réclame de Dieu lui-même, 2 / dont la prophétie est un appel à modifier la Torah, dans le but de servir Dieu. (Hannah Kasher, "Le-shinnui be-mashma'uto shel ha-kinnui 'ha-mitnabbe' be-shem 'abodah-zarah' be-mishnat ha-Rambam”, in. Maimonidean Studies (5), Arthur Hyman (éd.), New York, MSYU Press, 2008, p. 28.)

12. Le Livre de la connaissance, op. cit., pp. 97-98. Hilkhot yesodei ha-Torah, 9, 1.

13. Dans cet écrit polémique et apologétique, le christianisme et l'Islam sont tous deux qualifiés de «sectes des imitateurs de notre religion" (Épitres, op. cit., p. 62). Tous deux ont cherché à «modifier notre religion» (Ibid., p. 59). Jésus a ouvert la voie en annonçant « une religion en dehors de la Torah de Dieu et la diffusant en public parce que les deux Torah émaneraient de Dieu.» (Ibid., p. 57). Mahomet, qualifié de « dément » (Ibid., p. 58), se différencie de Jésus en tant qu'il chercha en outre à s'arroger un " pouvoir temporel » (Ibid., p. 59). Face à ces « imitateurs ", Maïmonide affirme que "cette Torah existe pour toujours» (Ibid., p. 79, je souligne). (Pour l'original arabe et une traduction hébraïque, cf. «Igeret Teman », in. Igrot ha-Rambam (1), Yi șhaq Shailat (éd.), Jérusalem, Ma'aleh Adumim, 1987, p. 77-168.)

14. Cf. Al-Fârâbi, Traité des opinions des habitants de la cité vertueuse, trad. Tahani Sabri, Paris, Vrin, 1990 et Kitab al-siyasa al-madaniya. The Political Regime, Beyrouth, Dâr el-masriq, 1993.

15. Rambam insiste lui-même sur le caractère absolument inédit de son ouvrage dans son introduction et le justifie par la dispersion du peuple juif, en mal d'unité en matière d'application de la Loi notamment.

16. Le Shulhan 'arukh par exemple, ouvrage du XVI siècle, resté jusqu'à aujourd'hui une référence majeure en matière de halakha, ne rapporte que les halakhot dont l'application est effective après la destruction du Temple (sur le modèle d'un décisionnaire prémaïmonidien, Yaakov ben Acher, dit «Ba'al ha-țurim»), bien que son auteur Yosef Karo s'appuie par ailleurs sur le Mishneh Torah, sur lequel il a écrit un commentaire.

17. Cette démarche n'est rien moins qu'évidente. Le Talmud (ТВ Sanhedrin 51 b et Zebahi:im 45a) semble présenter comme un principe que cela n'a ni sens, ni utilité de trancher la halakha dans des domaines qui n'ont pas cours en son propre temps (ce qu'énonce la question "hilkheta' limeshih ia'?", autrement dit "que vas-tu trancher la loi pour le messie?»). Ce n'est pas à dire que les lois inapplicables, par exemple celles qui concernent les sacrifices en l'absence de Temple, ne doivent pas être étudiées. Mais leur utilité réside précisément dans leur étude elle-même et non dans un arrêt juridique sans aucune portée pratique : drosh ve-qabbel sakhar, étudie et reçoisen le salaire (sur cette expression, cf. idem et surtout Sanhedrin 71a où certaines lois sont dites à la fois pratiquement inapplicables et avoir été données simplement pour être étudiées). Maïmonide semble renverser cette perspective : pour que l'étude des lois inapplicables soit bénéfique, il faut les envisager sur le mode du comme si : comme si elles avaient à être appliquées. Trancher comme le fait Maïmonide dans ces domaines c'est, semble-t-il, donner enjeu et consistance à leur étude. 
18. Guide, I, Introduction, p. 7 (je souligne).

19. Ibid., II, 36, p. 281.

20. Sur ce point, Maïmonide a d'ailleurs particulièrement influencé l'entreprise mystique d'Abraham Aboulafia. (cf. Idel, Moshe, Maïmonide et la mystique juive, trad. Charles Mopsik, Paris, Cerf, 1991, chpt. II, « Abraham Aboulafia et l'unio mystica »).

21. Guide, II, 38, p. 290.

22. Idem, p. 291.

23. Ibid., II, 32, p. 261-262. Maïmonide vise la prophétologie des péripatéticiens arabes, notamment d'Avicenne (voir la note 1 de S. Munk sur place).

24. Ibid., II, 38, p. 291.

25. «[...] Et tout le peuple voyait le tonnerre (Ex 20,15), - quoique, d'ailleurs, cette scène fût une vision prophétique. » (Ibid., I, 47, p. 161.)

26. "Quant à la scène du mont Sinaï, bien que tous (les enfants d'Israël), par la voie du miracle, vissent le grand feu et entendissent les sons redoutables et effrayants, il ne parvint pourtant au rang de la prophétie que ceux-là seuls qui y étaient propres, et cela à différents degrés. » (Ibid., II, 32, p. 267.)

27. Ibid., II, 33, p. 268.

28. La question de savoir si «Je suis l'Éternel ton Dieu » est un énoncé qui relève du domaine normatif du commandement (mișwah), est un grand sujet de polémique chez les commentateurs de Maïmonide. Naḥmanide notamment, dans son commentaire du premier commandement rapporté par Rambam dans son Livre des commandements (Sefer ha-mișwot), et Ḥasday Crescas, au début de son principal ouvrage, le Or Ha-Shem, le nient, arguant du fait qu'un tel commandement serait contradictoire. C'est le problème de la source de toute autorité normative, que l'on rencontre dans un autre contexte, en philosophie du droit. Si la capacité d'une instance à énoncer une norme repose sur une norme, il faut alors fonder la capacité à énoncer cette autre norme et ainsi de suite. Pour éviter une telle régression à l'infini, elle doit reposer soit sur un axiome ou une croyance qui attribue une telle capacité à l'instance normative en question, soit sur une «norme fondamentale ( (cf. Kelsen, Hans, Théorie pure du droit, trad. Charles Eisenmann, Paris, Dalloz, 1962), qui n'est pas elle-même fondée (du type « on doit obéir à Dieu » ou « il faut respecter les lois de l'État»). Qu'une autorité prescrive de croire en son autorité n'a en effet pas de sens, puisque c'est précisément parce qu'on y croit préalablement qu'on lui reconnaît le pouvoir de prescrire des normes.

29. Guide, II, 33, p. 269.

30. Ibid., II, 40, p. 310.

31. Idem, p. 311-312.

32. Idem, p. 311.

33. Ibid., III, 38, p. 214-216.

34. Ibid., III, 29, p. 230.

35. Idem.

36. Ibid., III, 32, p. 250.

37. Idem, p. 251.

38. Cf. Funkenstein, Amos, Maïmonide. Nature, histoire et messianisme, traduit de l'hébreu par Catherine Chalier, Paris, Cerf, 1988. L'idée directrice de cet ouvrage est que le concept « d'accommodation » est l'opérateur central de la pensée de Maïmonide. Dans tous les domaines, de la cosmologie à l'éthique, le Dieu de Maïmonide s'accommode des limitations inhérentes à sa création, en particulier du fait qu'elle implique nécessairement une part de contingence. Ce concept permet de rendre compte, notamment, d'une originalité de Maïmonide dans l'univers de l'aristotélisme arabe, à savoir son admission d'une part de contingence dans le monde supralunaire: il n'y a aucune raison par exemple pour que telle sphère se meuve dans telle 
direction plutôt que telle autre. Dieu, s'accommodant du fini, a dû choisir une direction plutôt qu'une autre, pour qu'un monde existe.

39. C'est la question dite des țáamei ha-mișwot, raisons ou significations (litt. goûts) des commandements. (Voir sur cette question l'ouvrage classique : Heinemann, Isaac, La loi dans la pensée juive, adaptation française Charles Touati, Paris, Albin Michel, 1962, p. 88-106.)

40. Le Livre de la connaissance, op. cit., p. 230 (traduction modifiée). Hilkhot 'abodah zarah, II, 2.

41. Initialement écrit en arabe, ce livre a été traduit en hébreu par Mosheh Ibn Tibbon, le fils du célèbre traducteur du Guide (Sefer ha-mișwot, in. Mishneh Torah (1), op. cit.). Nous citerons la traduction française établie à partir d'une traduction hébraïque ultérieure (Yosef Qapah) : Maïmonide, Le livre des commandements, trad. [de l'hébreu] Anne-Marie Geller, Paris, L'âge d'homme, 1987.

42. Le livre des commandements, op. cit., $10^{\mathrm{e}}$ commandement négatif, p. 207-208.

43. Guide, III, 29, p. 231.

44. «Le plus grand ouvrage sur ce sujet est l'Agriculture nabatéenne, ouvrage traduit par Ibn Waḥshiyya. ", (Idem et passim.)

45. Ibid., III, 46, p. 362 sq.

46. Le livre des commandements, loc. cit. traduction modifiée (je souligne), cf. le texte correspondant : Le Livre de la connaissance, op. cit., p. 230. Hilkhot 'abodah zarah, II, 2.

47. Cf. Ibid., p. 240. Hilkhot 'abodah zarah , III, 2.

48. Cf. Guide, I, Introduction, p. 25, n. 3.

49. Ibid., III, 51, p. 435.

50. Idem.

51. On trouvera en effet les jalons d'une telle position dans certains passages du volume : Strauss, Léo, Maïmonide, trad. Rémi Brague, Paris, PUF, 1988. Cf. notamment le texte intitulé «La philosophie et la loi ».

52. Sur la perfection de la Torah, comme Loi divine et son unicité : «... dès qu'une chose est la plus parfaite possible de son espèce, toute autre chose de la même espèce ne peut pas ne pas être moindre, soit en dépassant la juste mesure, soit en restant au-dessous. [...] Il en est de même de cette loi. » (Guide, II, 39, p. 303.)

53. «On ne se rase pas les coins de la chevelure à la façon qui était en usage chez les idolâtres et leurs prêtres. » (Le Livre de la connaissance, op. cit., p.339, traduction modifiée. Hilkhot 'abodah zarah, 12, 1). Dans son ouvrage classique sur le Mishneh Torah, Twersky indique d'autres références (Twersky, Isadore, Introduction to the Code of Maimonides (Mishneh Torah), New Heaven et Londres, Yale University Press, 1980, p. 431, n. 186). Sur la comparaison du traitement de la question des țáamei ha-mișwot dans le Guide et le Mishneh Torah, voir le chapitre VI de cet ouvrage,"Law and Philosophy".

54. Cf. Le Livre de la connaissance, op. cit., p. 405. Hilkhot teshubah, 7, 6.

55. «... la grandeur de ceux qui ont péché [et en sont revenus] est supérieure à celle des justes qui n'ont jamais péché, car ceux-là dominent leurs penchants plus que ceux-ci.» (Ibid., traduction modifiée, p. 404. Hilkhot teshubah, 7, 4).

56. Cf. Ibid., p. 408-414. Hilkhot teshubah, 8.

57. Ibid., p. 62. Hilkhot yesodei ha-Torah, 4, 8. Guide, I, 1-2.

58. Ibid., p. 64 (traduction modifiée). Hilkhot yesodei ha-Torah, 4, 9.

59. «Les temps messianiques ne sont rien d'autre que ce monde-ci (ha-'olam ha-ze'), sans modification de ses lois, à la différence près que la royauté d'Israël sera restaurée. » (Ibid., p. 419, traduction modifiée. Hilkhot teshubah, 9, 2, source : TB Sanhedrin 91b.)

60. La conception maïmonidienne des temps messianiques est exposée pour l'essentiel dans les derniers chapitres du Mishneh Torah, Hilkhot melakhim u-milhamot (règles relatives aux rois et à la guerre), 11-12. (Pour une vue synthétique de la question, cf. Aviezer Ravitzky, "To the Utmost of Human Capacity: Maimonides on the Days of Messiah", in Perspectives on Maimonides, Joel 
Kraemer (éd.), Oxford, Oxford University Press, 1991). Le traitement de la législation concernant les lois noachiques précède immédiatement celui des temps messianiques. Ainsi, en Hilkhot melakhim u-milhamot, 9, 11, Maïmonide tranche de manière étonnante que l'accès des non-juifs au monde à venir est réservé à ceux d'entre eux qui appliquent les sept lois noachiques parce qu'elles leurs ont été prescrites par Dieu dans la Torah et non parce qu'elles relèvent d'une législation rationnelle ou naturelle.

61. Le Livre de la connaissance, op. cit., p. 419 (traduction modifiée). Hilkhot teshubah, 9, 2.

62. «Mais le bonheur du monde à venir est incommensurable (ein lah 'erekh) et rien ne saurait lui être comparé de ce qui existe ici-bas, sinon par manière de métaphore.» (Ibid., p. 413. Hilkhot teshubah, 8, 6.)

63. Sur la " pédagogie » de Maïmonide, cf. «Introduction au chapitre Hélèq » (in. Épitres, op. cit., p. 162 sq.), où la promesse de récompense est expliquée comme un moyen pour inciter l'enfant à l'étude de la Torah, étude dont il est incapable de saisir l'intérêt propre.

64. Guide, III, 51, p. 436.

65. Idem, p. 438.

66. Idem, p. 442.

67. Idem, p. 444.

68. Ce qu'explicite très bien Léo Strauss, dans le texte d'une conférence non prononcée écrite en 1931 : «Parce qu'ils sont de fait sous la loi, [les philosophes à l'époque des religions révélées, dont Maïmonide] n'ont plus besoin comme Platon de chercher la loi, la Cité : l'ordre contraignant du vivre ensemble leur est donné par un prophète. Ils sont pour cette raison autorisés par la loi, libres pour la compréhension dans une liberté aristotélicienne. Ils peuvent par conséquent aristotéliser. » (Léo Strauss et Corine Pelluchon, « Cohen et Maïmonide », Revue de métaphysique et de morale (38), 2003, p. 273.)

69. «La plupart des commandements n'ont d'autre but que de nous faire arriver à cette espèce de perfection. Mais celle-ci n'est qu'une préparation à une autre perfection, et elle n'est pas une fin en elle-même. » (Ibid., III, 54, p. 461)

70. Ibid., III, 52, p. 453.

71. Idem, p. 454.

72. Le Livre de la connaissance, op. cit., pp. 35-36. Hilkhot yesodei ha-Torah, 2, 1-2.

73. Guide, III, 51, p. 444.

74. Ibid., III, 54, p. 459.

75. « ... toutes les créatures oublièrent en paroles et en pensée le Nom glorieux et terrible au point d'en ignorer [jusqu'à l'existence].» (Le livre de la connaissance, op. cit., p. 223, traduction modifiée. Hilkhot 'abodah zarah, 1, 2.)

76. Ibid., p. 230, traduction modifiée. Hilkhot 'abodah zarah, 2, 1.

77. Guide, I, 36, p. 136 (je souligne).

78. Entre autres : « ... le but principal de la Loi, c'est de faire cesser cette croyance et d'en effacer la trace... » (Guide., III, 30, p. 246) ; «... le but et pour ainsi dire le centre de la Loi, c'était la destruction de l'idolâtrie, dont elle voulait faire disparaître la trace... » (Ibid., III, 37, p. 280).

79. À moins de donner à une telle conception une formulation dialectique, à la manière de $Y$. Leibowitz: "La halakhah, de prime abord, perçue comme l'ensemble des détails du service de Dieu, apparaît comme un aspect extérieur de la religion, comme une sorte d'enveloppe protectrice du noyau qui est la croyance en Dieu. [...] Néanmoins, à l'échelon supérieur, on découvre que - lorsque l'homme perce l'enveloppe en direction du noyau et arrive à cette foi en Dieu qui consiste en l'éradication absolue de toute idolâtrie - ses yeux se dessillent et il se rend à l'évidence que ce qu'il prenait au début pour une enveloppe extérieure de la religion n'est, en fait, que son contenu véritable. " (Leibowitz, Yeshayahu, La foi de Maïmonide, trad. David Banon, Paris, Cerf, 2004, p. 34). Autrement dit, ce que Leibowitz nomme « service désintéressé de Dieu ", qui traduit l'expression Torah li-shemah, littéralement la Torah pour elle-même, en tant que fin en 
soi et non comme moyen, n'échappe à l'idolâtrie qu'au terme du procès dialectique qui interroge le noyau, le fondement et le sens mêmes du culte.

80. Cf. supra.

81. Guide, III, 29, pp. 238-239.

82. Idem, p. 235 (je souligne).

83. Le rapprochement esquissé ici mériterait d'être approfondi. Il n'est cependant pas arbitraire puisque Marx lui-même en pose les jalons: «Si bien que pour trouver une analogie [avec le monde marchand], nous devons nous échapper vers les zones nébuleuses du monde religieux. Dans ce monde-là, les produits du cerveau humain semblent être des figures autonomes, douées d'une vie propre, entretenant des rapports les unes avec les autres et avec les humains. » (Marx, Karl, Le Capital, I, 1, 4, «Le caractère fétiche de la marchandise et son secret ", Jean-Pierre Lefebvre (dir.), Paris, PUF, 1993, p. 83). Les individus qui se rencontrent sur le marché attribuent aux marchandises une autonomie vis-à-vis des rapports sociaux dont pourtant ils résultent. Le marché en d'autres termes est un système autorégulé de manière purement immanente. À moins d'être marxiste, l'individu ne sait pas ce qu'il fait lorsqu'il manie des marchandises. La forme marchandise s'érige en quelque sorte entre l'individu et le social comme le culte idolâtrique entre l'homme et Dieu. (Voir aussi Balibar, Étienne, La philosophie de Marx, Paris, La découverte, 1993, pp. 41-74.) Erich Fromm a par ailleurs formulé à partir de sources juives une critique d'inspiration marxiste de l'idolâtrie moderne y incluant outre le culte de la production et de la consommation, celui de l'État et de ses dirigeants (Fromm, Erich, Vous serez comme des dieux, trad. Paul Alexandre, Paris, Complexe, 1975 [1966], pp. 43-52 et passim).

84. Guide, III, 26, p. 209.

85. Cf. Funkenstein, Amos, op. cit., chapitres III et IV.

86. Le livre des commandements, op. cit., $187^{\mathrm{e}}$ commandement positif, p. 171. Sefer ha-miswot, op. cit., p. 273.

87. «Demander alors une pareille chose [l'abolition immédiate des sacrifices], c'eût été comme si un prophète dans ces temps-ci, en exhortant au culte de Dieu, venait nous dire: "Dieu vous défend de lui adresser des prières, de jeûner, et d'invoquer son secours dans le malheur; mais votre culte sera une simple méditation sans aucune pratique." » (Guide, III, 32, p. 251)

88. Mishneh Torah, Hilkhot melakhim u-milhamot, 11, 10.

89. «Pour comprendre le messianisme juif », in Scholem, Gershom, Le messianisme juif. Essais sur la spiritualité du judaïsme, trad. Bernard Dupuy, Paris, Calmann-Levy, 1974.

90. Idem.

91. Épitres, op. cit., pp. 95-97. (Igrot ha-Rambam (1), op. cit., pp. 105-106 et trad. pp. 152-153.)

92. Nous nous écartons ici de l'interprétation proposée par Funkenstein du caractère restaurateur du messianisme maïmonidien. Selon Funkenstein, en effet, la perspective de la restauration du Temple révèle le peu de confiance de Maïmonide dans la nature humaine. Il est toujours à craindre que les esprits faibles ne soient pas à même d'accéder à la connaissance de Dieu que permettront les temps messianiques et ne retombent dans leurs anciennes erreurs (c'est-à-dire les anciennes formes de l'idolâtrie). D'où la nécessité d'un culte sacrificiel. (Cf. Funkenstein, Amos, op. cit., p. 79 et passim.). En revanche, nous sommes partiellement d'accord avec celle d'Howard Kreisel, selon laquelle la préoccupation principale de Maïmonide dans sa doctrine du sens des commandements est sa propre époque et non un passé ou un futur idéalisé (Howard Kreisel, "Reasons for the Commandments in Maimonides' Guide of the Perplexed and in Provencial Jewish Philosophy", in Maimonidean Studies (5), op.cit., p. 183). Autrement dit, son messianisme restaurateur ne vise qu'à conférer sens et vigueur à la Torah au présent. 


\section{RÉSUMÉS}

In his Mishneh Torah, Maimonides insists on the immutability of the Torah and describes the messianic era as a time when it will be completely restored. On the contrary, some passages from his Guide of the perplexed seem to draw a conception of the Mitswot (commandments) according to which they are determined, as far as their content is concerned, by the general human behaviours at the time of the revelation of the Torah. In other words, once these specific behaviours disappeared, the commandments became obsolete. In this study, I try to reconcile these two theses, by suggesting that this very contradiction hints at a unitary and original conception of Law, temporarily inapplicable in its entirety, yet absolutely effective and significant at all time, appropriate thus for its principal aim: combating a perverted rapport with the world and with the laws, which is referred to as idolatry.

INDEX

\section{מילות מפתח}

מורה נבוכים, הרמב"ם, בספרות חז"ל, ההיסטוריות, פילוסופיה:

Keywords : Maïmonide Moïse (1135-1204), Guide of The Perplexed, rabbinic literature, philosophy, historicity

Mots-clés : Maïmonide Moïse (1135-1204), Guide des égarés, littérature rabbinique, historicité

Thèmes : philosophie 